\title{
An algorithm for operational flood mapping from Synthetic Aperture Radar (SAR) data using fuzzy logic
}

\author{
L. Pulvirenti ${ }^{1}$, N. Pierdicca ${ }^{1}$, M. Chini ${ }^{2}$, and L. Guerriero ${ }^{3}$ \\ ${ }^{1}$ Department of Information Engineering, Electronics and Telecommunications, Sapienza University of Rome, \\ Via Eudossiana 18, 00184 Rome, Italy \\ ${ }^{2}$ Istituto Nazionale di Geofisica e Vulcanologia, Via di Vigna Murata 605, 00143 Rome, Italy \\ ${ }^{3}$ Department of Computer Science, Systems and Production, Tor Vergata University of Rome, Via del Politecnico 1, \\ 00133 Rome, Italy
}

Received: 13 July 2010 - Revised: 9 December 2010 - Accepted: 4 January 2011 - Published: 18 February 2011

\begin{abstract}
An algorithm developed to map flooded areas from synthetic aperture radar imagery is presented in this paper. It is conceived to be inserted in the operational flood management system of the Italian Civil Protection and can be used in an almost automatic mode or in an interactive mode, depending on the user's needs. The approach is based on the fuzzy logic that is used to integrate theoretical knowledge about the radar return from inundated areas taken into account by means of three electromagnetic scattering models, with simple hydraulic considerations and contextual information. This integration aims at allowing a user to cope with situations, such as the presence of vegetation in the flooded area, in which inundation mapping from satellite radars represents a difficult task. The algorithm is designed to work with radar data at $\mathrm{L}, \mathrm{C}$, and $\mathrm{X}$ frequency bands and employs also ancillary data, such as a land cover map and a digital elevation model. The flood mapping procedure is tested on an inundation that occurred in Albania on January 2010 using COSMO-SkyMed very high resolution X-band SAR data.
\end{abstract}

\section{Introduction}

Floods are probably the most frequent, disastrous and widespread natural hazards of the world. Mapping their extension is fundamental to assess the damages and for relief organization. The potential of the data provided by satellite Synthetic Aperture Radar (SAR) systems for large-scale flood detection has been demonstrated by several previous investigations (e.g., Horritt et al., 2001). The synoptic view and the capability to operate during daytime and nighttime and

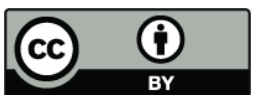

Correspondence to: $\mathrm{L}$. Pulvirenti (pulvirenti@die.uniroma1.it) in almost all weather conditions, contrary to visible/infrared sensors, are the key features that make SAR images useful for inundation mapping (Smith, 1997). In addition, the great sensitivity to water of the microwave band permits SAR to distinguish between land and water.

Several inundation events were profitably analyzed in the past by using SAR imagery. Examples are the Mississippi flood of 1993 (Brakenridge et al., 1994), the 1996 and 1997 inundations in the Red River Valley (Barber et al., 1996; Wilson and Rashid, 2005), the August 2002 Elbe river flood (Henry et al., 2006), the overflow of the River Thames in 1992 (Horritt et al., 2001), the 2006 event of the River Dee in Wales (Schumann et al., 2009a), the River Mosel flood of 1997 and the 2003 event on the River Alzette floodplain (Schumann et al., 2007). Reviews of the state of the art in flood remote sensing were provided by Smith (1997), Sanyal and Lu (2004) and by Schumann et al. (2009b).

Few investigations on the operational use of SAR data for inundation mapping are available in literature, mainly because of the fairly long temporal repetitiveness of SAR measurements, which has been a critical issue so far. An unsupervised method for near real-time flood detection using SAR data has been recently proposed by Martinis et al. (2009) and tested on a TerraSAR-X scene acquired throughout heavy floods occurring at the River Severn (UK) in 2007. The data provided by the COSMO-SkyMed (COnstellation of small Satellites for Mediterranean basin Observation) mission represent a powerful tool for operational flood management. COSMO-SkyMed (the acronym CSK will be also used hereafter) is a cooperation project between the Italian Space Agency (ASI) and the Italian Defence Ministry which has been developed to provide users with fast, meter level-resolution, all-weather X-band SAR data for disaster management. With the full constellation of four satellites

Published by Copernicus Publications on behalf of the European Geosciences Union. 
deployed (the fourth satellite was launched on 5 November 2010), the revisit time will be $12 \mathrm{~h}$ in the worst case (Covello et al., 2010).

ASI is presently funding some studies on the use of Earth Observation data for managing flood events. Within the framework of one of these investigations (OPERA, civil protection from floods), whose purpose is the evaluation of the usefulness of Earth Observation techniques in operational flood prediction and assessment chains (Boni et al., 2009), we have developed a new algorithm to produce maps of inundated areas from SAR images. It has been conceived to be used for operational flood management system of the Italian Civil Protection.

The algorithm is based on fuzzy logic that is particularly suitable to deal with the ambiguities in radar images (Karmakar and Dooley, 2002). Moreover, fuzzy logic has permitted us to take into account different information sources, in particular: (i) the outputs of three electromagnetic scattering models used to simulate the SAR observations of flooded areas; (ii) simple hydraulic considerations based on the nearness to water bodies (rivers, lakes, reservoirs, etc.) and on the height of the analyzed pixels; (iii) contextual conditions relating neighbouring pixels.

The validity of an approach based on fuzzy logic for flood mapping was assessed in Pierdicca et al. (2008), where the parameters of the fuzzy algorithm were selected, taking advantage of the availability of ground truth data concerning the flood extension. Since in operative applications such availability cannot be supposed, in the present work we follow a different method. The theoretical knowledge on the scattering of the electromagnetic radiation from various types of surfaces, that in Pulvirenti et al. (2011) proved to be very useful for flood mapping using SAR, is exploited. In particular, three electromagnetic models predicting the radar return from agricultural, forested and urban areas, have been extended to simulate the backscattering under flooded conditions. Note that in Pierdicca et al. (2008), the nearness to water bodies and the height of the pixels were not considered. Furthermore, here we also account for the homogeneity of the radar return from open water surfaces.

The algorithm allows the user to perform an almost automatic generation of a map of flooded areas. In this case, the default parameters of the algorithm, i.e., those derived from the outputs of the theoretical models (see Sect. 2.1) are retained. Alternatively, expert operators can interactively modify the parameters of the algorithm. For this purpose, they can rely on their visual interpretation of the SAR imagery (Schumann et al., 2009a), but they can also analyze the statistics of the backscattering from some regions of interest (ROIs) that can be drawn on the images. In other words, both fairly accurate flood boundary delineation, based on a large amount of user interaction, and an automatic rapid mapping are allowed in order to account for the different needs of Civil Protection in the different stages of its operational tasks.
Section 2 introduces the fuzzy approach that we have used to design the algorithm and points out its advantages with respect to other image- processing techniques used to separate inundated from non-inundated areas in SAR images. The algorithm is described in detail in Sect. 3, while its application to a test case is discussed in Sect. 4. Section 5 draws the main conclusions.

\section{The fuzzy approach}

Although both coherence change detection methods (e.g., Nico et al., 2000) and active contour models (e.g., Horritt et al., 2001) have been frequently used in literature to detect flooded areas in SAR imagery, thresholding represents the most common technique for inundation mapping from radar data. According to this approach, all pixels of an intensity image whose backscattering coefficient $\left(\sigma^{0}\right)$ is smaller than a given threshold value (e.g., Hess et al., 1995; Henry et al., 2006) are classified as flooded. Thresholding is computationally inexpensive and suitable for rapid mapping purposes (Martinis et al., 2009), but is valid only for open water surfaces that behave as specular reflectors, thus scattering a small amount of radiation back to the radar antenna.

In vegetated, forested, or urban areas, flooded conditions may cause an increase of the radar return, because of the enhancement of the double bounce backscattering mechanism, which involves the specular water surface and vertical structures, such as stems, trunks (Hess et al., 1990), or buildings. This increase, whose magnitude depends on the SAR parameters (frequency band, polarization and observation angle), may be detected by applying a threshold to the pixel by pixel difference between the $\sigma^{0}$ of the radar observation of the flood (hereafter denoted also as image-1) and the $\sigma^{0}$ of a SAR image of the considered geographical area under dry conditions (hereafter image-2) (Pulvirenti et al., 2010). The difference between the radar intensities can be computed considering both linear and $\mathrm{dB}$ (i.e., logarithmic) units, although the use of the latter is generally recommended because a difference in $\mathrm{dB}$ units corresponds to apply the ratio operator that was shown to be better adapted to the statistical characteristics of SAR data (e.g. Rignot and van Zyl, 1993).

The dependence of the radar echo from flooded pixels on land cover, system parameters and environmental conditions implies that the thresholds are generally determined through a supervised approach, i.e., a visual inspection of the SAR images. Martinis et al. (2009) stated that the major drawback of this methodology is that the reliability of the final result strongly depends on the skill of the operator, so that they proposed a completely unsupervised algorithm based on an automatic thresholding approach that did not take advantage of any training data or prior information. Conversely, Mason et al. (2010) claimed that supervised methods with large amount of user interaction are required to perform a reliable inundation mapping from SAR data, especially if urban areas 
are included in the imaged scene. A way to deal with the ambiguity of the radar signature of flooded areas and, at the same time, with the requirement of an objective result has been implemented in our algorithm, based on the fuzzy theory. Exploiting prior information on the physics of surface scattering, the algorithm can perform an automatic mapping, but it can also allow a certain degree of user interaction when considered relevant for the specific scenario.

The fuzzy sets basically represent an extension of the classical notion of set. While in classical set theory an element either belongs or does not belong to the set, elements of a fuzzy set have degrees of membership. These degrees are defined through membership functions whose values are real numbers in the interval $[0,1]$. Examples of simple membership functions are the standard $S$ function and the standard $Z$ function (Pal and Rosenfeld, 1988) that are shown in Fig. 1, in which the parameters $x_{1}$ and $x_{2}$ denote the fuzzy thresholds. The fuzzy theory is suitable for representing the set of flooded pixels in SAR images for which the definition of a criterion of membership is a difficult task.

\subsection{The model-based fuzzy thresholds}

In order to avoid resorting to empirical training data sets generally unavailable in operative applications, we have determined the default values of the fuzzy thresholds by analyzing the outputs of three well-established electromagnetic scattering models, namely the two models developed at the Tor Vergata University of Rome (Bracaglia et al., 1995; Ferrazzoli and Guerriero, 1995) to simulate the radar return from agricultural and forested areas, and that proposed by Franceschetti et al. (2002) to predict the backscattering from urban areas. They have been run for different system parameters, such as frequency bands $(\mathrm{L}, \mathrm{C}, \mathrm{X})$, polarizations (horizontal and vertical, i.e. only co-polarized data are considered), and incidence angles $\left(25^{\circ}, 35^{\circ}\right.$ for $\mathrm{L}$ and $\mathrm{C}$ bands; $25^{\circ}, 35^{\circ}, 45^{\circ}, 55^{\circ}$ for $\mathrm{X}$ band, accounting for the multi-angle capability of CSK). Sixteen files of default threshold values (2 polarizations times 2 angles for $\mathrm{L}$ and $\mathrm{C}$ bands; 2 polarizations times 4 angles for $\mathrm{X}$ band) have been produced. Each file contains the thresholds for four land cover classes: bare soils, urban or built-up areas, agricultural terrains and forests, so that the availability of a land cover map (LCM) is supposed.

Geometrical and dielectric properties assumed for modelling the scattering of vegetation have been based on the most common species in the Mediterranean environment. As for agricultural areas, we have considered typical characteristics (e.g., leaf and stem dimensions, plant height and density) of crops (Ferrazzoli et al., 2000; Della Vecchia et al., 2006), whereas for forests, biomasses of $25 \mathrm{tha}^{-1}$ and $50 \mathrm{tha}^{-1}$ have been considered. Note that a more accurate discrimination of vegetation types than a simple distinction between crops and forests would have required the use of detailed maps, generally unavailable in operative applications.
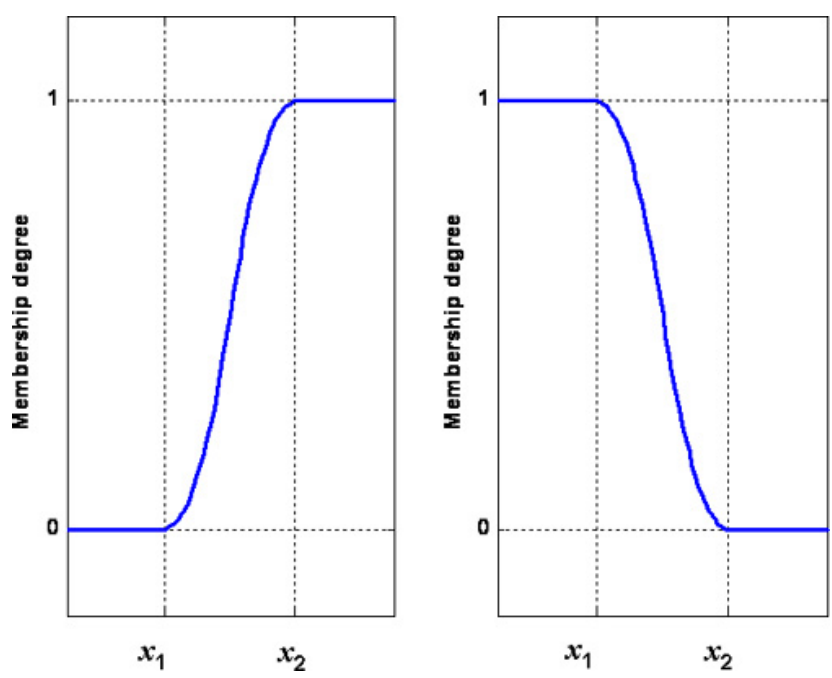

Fig. 1. Standard $S$ (left panel) and $Z$ (right panel) fuzzy membership functions. For the $\mathrm{S}$-function, values larger than $x_{2}$ belong definitely to the set (membership degree equal to 1 ), while values smaller than $x_{1}$ do not belong to the set (membership degree equal to 0 ). For the Z-function, the opposite occurs.

Moreover, dealing with many different vegetation species would have increased the complexity of the models of electromagnetic scattering of vegetated areas considerably. We have therefore made a compromise between the quality of the predictions of the backscattering coefficient and the complexity of the designed system, also accounting for the expected quality of the information about land cover.

To run the models, the flood conditions have been simulated by assuming that soil has the same electromagnetic characteristics (i.e., dielectric permittivity) as water and a negligible roughness (Pulvirenti et al., 2011) as well. To predict the radar return under dry conditions, fairly standard values of soil roughness (i.e., height standard deviation in the order of $1 \mathrm{~cm}$ ) and volumetric moisture (in the range $0.2-$ $0.3 \mathrm{~m}^{3} \mathrm{~m}^{-3}$ ) have been assumed.

Simulated radar returns from agricultural areas have been produced by considering, as crops, wheat and corn at different stages of growth (from bare soils to well-developed vegetation). By analyzing the simulations, we have found that, under flooded conditions, plants at an early stage of growth show a $\sigma^{0}$ similar to that of bare soils (almost specular reflection of the radar radiation). Also, developed plants submerged by water (i.e., water level higher than crop height) tend to specularly reflect an electromagnetic wave.

We have defined the fuzzy set of low backscattering areas, whose degree of membership is evaluated by applying the standard Z-function (Fig. 1, right panel) to image-1. If $\sigma^{0}<x_{1}$ the degree of membership to the set of inundated pixels is 1 , while if $\sigma^{0}>x_{2}$ it is 0 . Note that $\sigma^{0}$ is in $\mathrm{dB}$ units. To determine $x_{1}$ and $x_{2}$ for the set of low backscattering areas, we have considered the outputs of the model of 
Table 1. Default fuzzy thresholds derived from the electromagnetic models for $\mathrm{X}$ band, incidence angle equal to $35^{\circ}$, horizontal polarization.

\begin{tabular}{lcc}
\hline Class & $x_{1}[\mathrm{~dB}]$ & $x_{2}[\mathrm{~dB}]$ \\
\hline Low backscattering areas & -19 & -10 \\
Agricultural terrains & 2 & 4 \\
Urban areas & 4 & 6 \\
Forests & 3 & 5 \\
\hline
\end{tabular}

agricultural areas obtained for bare soil and for small crops. Furthermore, for some SAR configurations ( $\mathrm{C}$ band, vertical polarization; $\mathrm{X}$ band, horizontal polarization) we have also exploited a set of SAR images (in particular, ERS, ENVISAT/ASAR and COSMO-SkyMed ones) belonging to our archive and including water bodies, easily detectable thanks to the sensitivity of the microwave band to water. The radar observations over water bodies have been used to tune the results of the theoretical model.

For agricultural areas, the simulations have shown that the increase of the double bounce backscattering due to an inundation can become quite large (at least $2-3 \mathrm{~dB}$ ) when the crops have reached at least an intermediate stage of growth (i.e., in spring or in summer). This effect is particularly evident for wheat, and for the $\mathrm{C}$ and $\mathrm{X}$ bands (Pulvirenti et al., 2011). The degree of membership to the class of agricultural flooded areas involved in the enhancement of backscattering is defined by the standard S-function (Fig. 1, left panel). This means that denoting as $\Delta \sigma^{0}$ the difference (in $\mathrm{dB}$ units) between the radar returns of image- 1 and image- 2 , if $\Delta \sigma^{0}<x_{1}$ the degree of membership to the set of inundated pixels is 0 , while if $\Delta \sigma^{0}>x_{2}$ the degree is 1 . The values of $x_{1}$ and $x_{2}$ have been determined through a joint analysis of the results obtained for wheat and corn. The S-function has been also used to define the membership to the sets of flooded forested and urban areas for which a backscattering enhancement may occur as well.

The electromagnetic model for forests has been run for both deciduous and coniferous forests and two biomasses $\left(25 \mathrm{tha}^{-1}\right.$ and $\left.50 \mathrm{tha}^{-1}\right)$. By analyzing the results of the simulations, we have found that, under flooded conditions, the two kinds of forest show approximately the same electromagnetic behaviour (i.e., the enhancement of the backscattering due to the double bounce is of the same order of magnitude). In addition, the increase of the backscattering has proved to be fairly large at horizontal polarization and for small biomasses. It is worth noting that, for some configurations of SAR parameters, agricultural and forested areas have not shown any sensitivity to water coverage, according to the model developed at the Tor Vergata University. For instance, at vertical polarization, the detection of flooded forests has turned out to be problematic at any bands. This finding sup- ports our idea of using fuzzy logic also to integrate hydraulic considerations into the algorithm (see Sect. 3), in order to improve its ability to map an inundation.

As for urban areas, relating the radar response patterns to the characteristics of the man-made structures is a very complex problem, whose solution is far to be found. This consideration implies that, as stated by Schumann et al. (2009b), SAR is unable to extract flooding from urban areas, even though an attempt has been recently carried out by exploiting the high resolution TerraSAR-X instrument (Mason et al., 2010). An analytical evaluation in closed form of the electromagnetic return from a building was accomplished by Franceschetti et al. (2002) for the simplified scenario represented by a building isolated from other man-made structures, i.e., a parallelepiped placed on a rough surface. Our simulations of radar return from urban flooded areas have been performed by applying the model of Franceschetti et al. (2002) substituting the rough surface surrounding the building with water. The results have revealed that the presence of the standing water causes a large increase of the double bounce backscattering, involving surface and buildings, at all the considered frequencies.

Table 1 reports the values of the default fuzzy thresholds that we have derived for $\mathrm{X}$ band, horizontal polarization, at incidence angle $\theta=35^{\circ}$. It is worth pointing out that, for agricultural areas and forests, the models provide a range of predicted backscattering coefficients under flooded conditions because of the different assumptions of the type and of the stage of growth of the crops or of the forest biomass. This range defines the values of $x_{1}$ and $x_{2}$. For instance, we have found that, for the sensor configuration considered in Table 1, the minimum increase (when present) of $\sigma^{0}$ due to flood for the class of agricultural areas, considering plants reaching at least an intermediate stage of growth, is $2 \mathrm{~dB}$, while the maximum increase can reach $4-5 \mathrm{~dB}$. These values correspond to those reported in Table 1. It can be noted that the values of $x_{1}$ and $x_{2}$ for the class of urban environment are larger than those for agricultural/forested terrains, in agreement with the previous considerations about the strong double bounce occurring in urban settlements.

We trust that the application of the fuzzy theory, which intrinsically accounts for the uncertainties in determining a threshold value separating flooded and non-flooded pixels, and the use of electromagnetic scattering models in order to determine the default values of the fuzzy parameters, can represent a valuable way to tackle the problem of the dependence of the outputs of the algorithm on the visual interpretation of the operator. However, the intervention of a skilled user can become important in particular environmental conditions. Indeed, the default thresholds are those expected in a mid-latitude area (we point out again that the software is conceived to be used by the Italian Civil Protection) in fairly standard situations. These situations can be schematically represented by absence of high wind speed, agricultural areas in which crops are mainly cereals, or in which the values of 
the vegetation parameters (height, biomass, etc.) are similar to those typical of cereals (see for instance Ferrazzoli et al., 2000), and by forests with moderate biomass. Different environmental conditions can be encountered, and in this case, the importance of the user interaction increases. For instance, it is likely the thresholds of the set of low backscattering pixels need to be modified if the wind speed is high because the wind roughening implies that open water surfaces do not behave as specular reflectors. Moreover, if different climatic areas are considered, vegetation parameters might be very dissimilar with respect to those assumed to run the electromagnetic models and the fuzzy thresholds may require an adjustment. User interaction is also important when dealing with urban areas, as stated in the literature (e.g. Mason et al., 2010).

\subsection{The fuzzy sets based on texture, context and ancillary data}

Fuzzy logic has also allowed us to include additional sources of data in the classification algorithm in order to improve the reliability of its results. Indeed, together with the set of flooded pixels according to SAR data, other fuzzy sets are generated by the algorithm. For each image pixel, the membership to the following fuzzy sets is defined: (A) set of homogeneous areas, i.e., of the areas in which the standard deviation of the backscattering is small; (B) set of pixels close to permanent water bodies; (C) set of pixels located at an altitude not exceeding that of the nearest permanent water body; (D) set of pixels surrounded by a uniform background; (E) set of pixels whose altitude is lower with respect to the elevation of the neighbouring ones that have a large degree of membership to the set of flooded areas; (F) set of pixels whose altitude is higher with respect to the elevation of the neighbouring ones that have a small degree of membership to the set of flooded areas.

Set $(A)$ is built through the application of a $3 \times 3$-pixel window scanning image- 1 and through the computation of the standard deviation of the radar return in the window. Then, the Z-function is applied to the image formed by the standard deviations with $x_{1}=0, x_{2}=0.1$. In this way, the degree of membership is close to 1 if the standard deviation is close to 0 , so that we assign a large membership degree to the set of flooded pixels to homogeneous zones. Indeed, in SAR images affected by the speckle noise, the standard deviation of the radar return is proportional to the mean value, so that open water surfaces generally appear as homogeneous areas (except when high winds roughen the surface).

As for sets (B) and (C), the basic idea is that if a river overflows, the inundation probability is large for pixels close to the river and at an altitude not exceeding the river course, so that these pixels should have a large degree of membership to the set of flooded areas. In this way, we avoid false alarms in areas far from water bodies and in hilly or mountainous regions. For each land pixel, the program can calculate its distance from the closest water body pixel, as well as the difference between the elevations of the two pixels. To create two fuzzy sets to account for these hydraulic considerations in the algorithm, the Z-function is applied and the parameters $x_{1}$ and $x_{2}$ are chosen equal to $0 \mathrm{~m}$ and $900 \mathrm{~m}$, respectively, for the distance from the water bodies, and to $0 \mathrm{~m}$ and $100 \mathrm{~m}$, respectively, for the height difference. Ancillary data are needed to build the sets (B) and (C). In particular, a LCM and a digital elevation model (DEM) are used. LCM should discriminate also water bodies, besides the four classes (urban areas, bare soils, agricultural terrains and forests) listed in Sect. 2.1.

The fuzzy sets (D), (E), (F) are built to account for contextual information in our algorithm, in order to avoid the presence of either isolated inundated pixels or small holes inside an inundated area, thus producing a final map having fairly large spatial coherence. In particular, pixels surrounded by a uniform background tend to assume the degree of membership of the background, while set (E) has a large degree of membership to the set of flooded areas and the opposite occurs for set $(F)$. To generate these fuzzy sets, we basically use the same membership functions that we developed in Pierdicca et al. (2008).

It is worth noting that the effect of introducing the aforementioned sets may depend on the quality of the ancillary data. For instance, sets $(\mathrm{E})$ and $(\mathrm{F})$ are almost ineffective if a low quality DEM is used. Furthermore, we expect to update the fuzzy thresholds of the sets based on texture, context and ancillary data on the basis of the analysis of other case studies. The thresholds we have used in this work have been also based on past experiences (e.g. Pierdicca et al., 2008).

As will be described hereafter, the various fuzzy sets are combined through the operation of fuzzy union (degree of membership defined as the maximum of the individual degrees) and through a fuzzy rule that defines the membership degree as the weighted average of the individual degrees (Karmakar and Dooley, 2002). The latter rule will be denoted hereafter as the weighted average rule.

\section{Algorithm description}

The flow diagram shown in Fig. 2 describes the structure of the algorithm that we have designed. On the left side of the diagram (in red), the operations that the user performs are represented, while on the right side (cyan), we indicate what the program displays during its run.

\subsection{The input data}

After the program is started, the user is asked to enter the SAR parameters (band, polarization, observation angle) that are used by the algorithm to select the appropriate file that contains the default fuzzy thresholds (see Sect. 2). Note that, for each of the considered frequency bands, the thresholds 


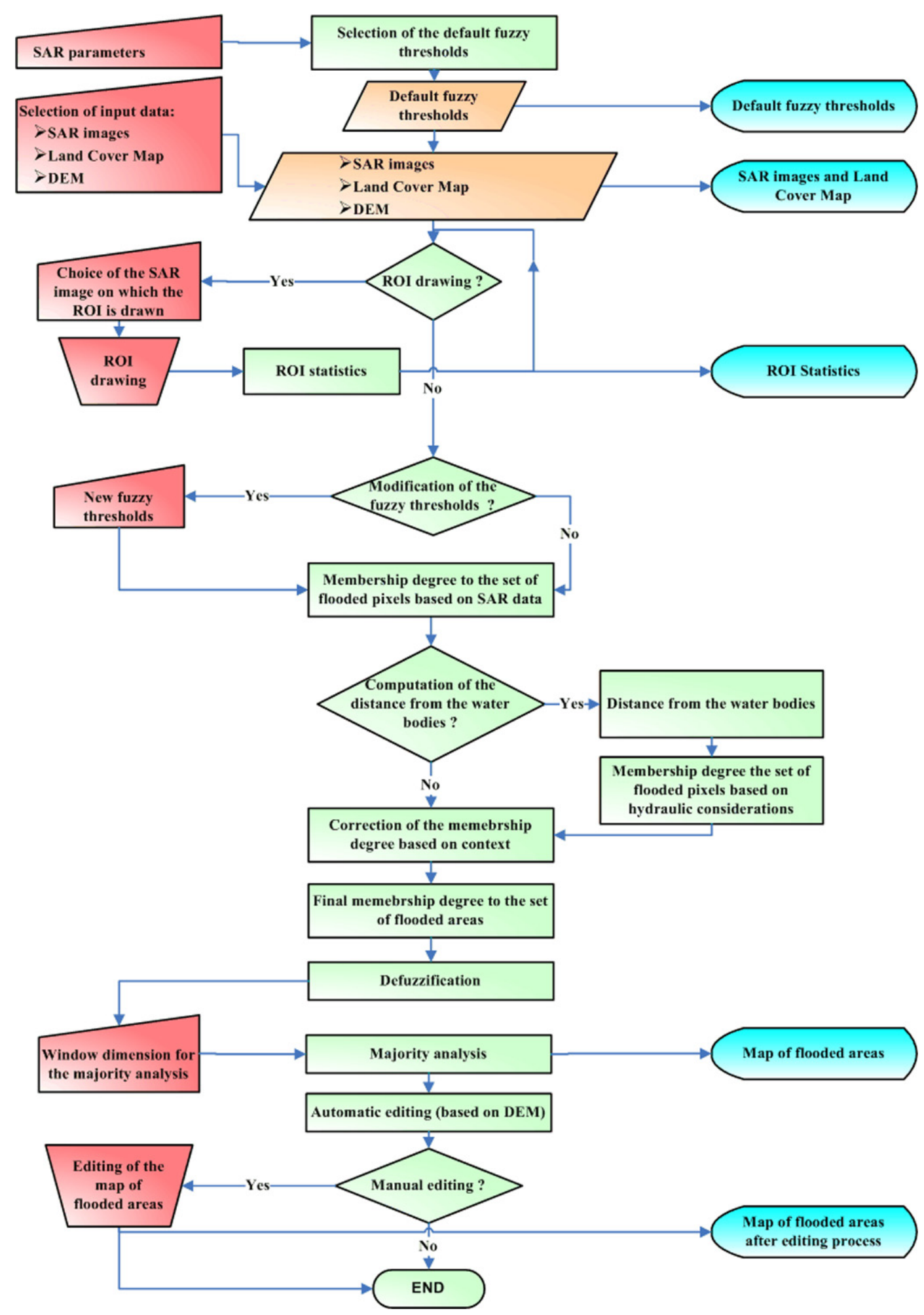

Fig. 2. Flow diagram of the designed algorithm. On the left part of the diagram, the user operations are indicated and represented by red trapeziums (manual inputs) and red isosceles trapeziums (manual operations). On the central part of the diagram, the various steps performed by the algorithm are represented by orange parallelograms (input data reading and storage), green rectangles (processes and operations) and green diamonds (decisions). On the left part of the diagram, the data displayed to the user are indicated (cyan forms). 
have been determined for a fixed number of incidence angles, so that, if the incidence angle of the SAR image is different, the program performs an interpolation to compute the default fuzzy threshold values.

Successively, the input data should be selected. The algorithm requires as inputs a SAR observation (intensity image) of the flooded area (image-1), a LCM and a DEM. A SAR observation of the same area under dry conditions (image2), helpful to detect vegetated and urban inundated regions (see Sect. 2), is an optional input, so that the program runs also if only image-1 is available. SAR data, LCM and DEM are supposed to be co-registered on the same reference cartographic system and must have the same pixel size and the same dimensions (i.e., number of rows and columns). The algorithm produces an output consisting of a map of inundated areas represented by a raster image having the same characteristics (reference cartographic system, pixel size, number of rows and columns) of the input data.

It must be considered that, if data provided by instruments with multi-incidence angle capability (such as COSMOSkyMed) are employed it may happen that image-1 and image- 2 have different incidence angles. Such a difference must be accounted for when evaluating the possible enhancement of the double bounce backscattering due to the inundation. We have assumed a simplified cosine model (Ulaby and Dobson, 1989), supposing that the variation of $\sigma^{0}$ with the incidence angle $\theta$ can be approximated by $\cos ^{2}(\theta)$. With this hypothesis, if $\theta_{\mathrm{x}}$ and $\theta_{\mathrm{y}}$ are the incidence angles of image- 1 and image-2, respectively, the quantity

$D=10 \log _{10}\left[\cos ^{2}\left(\theta_{\mathrm{x}}\right)\right]-10 \log _{10}\left[\cos ^{2}\left(\theta_{\mathrm{y}}\right)\right][\mathrm{dB}]$

is added to image- 2 and angle $\theta_{\mathrm{x}}$ is considered to select the fuzzy thresholds.

It is worth noting that, for rapid mapping purposes, the operator could decide to use only the radar observation of the flood, in order to avoid losing time searching through a data archive for a SAR image of the area involved in the flood event under dry conditions (provided by the same radar, preferably with the same observation geometry).

\subsection{Region of interest drawing and modification of the fuzzy thresholds}

As discussed at the end of Sect. 2.1, the default fuzzy thresholds have been established by assuming fairly standard conditions of a mid-latitude region, such as the Mediterranean, for the runs of the electromagnetic scattering models. Since different environmental conditions can be encountered, the operator can verify whether the default thresholds are suitable for the specific case study and, if necessary, interactively change them. For this purpose, the algorithm allows the operator to compare the default thresholds with the radar return from one or more ROIs that can be drawn on the SAR images.
Once the input data have been entered, the program displays the default fuzzy thresholds $\left(x_{1}\right.$ and $\left.x_{2}\right)$ together with the SAR images (i.e., image- 1 and, in case, image- 2 and the pixel by pixel difference, in $\mathrm{dB}$ units, between image- 1 ad image-2) and the LCM. Then, the user can choose the image on which he/she wishes to create the ROI and, after the ROI has been drawn, the program displays the statistics (mean value and standard deviation) of the radar return inside the ROI for all the available SAR images. To select the ROI on homogeneous regions from a land cover point of view, the operator can help himself/herself by looking at the displayed LCM. The operation of ROI drawing can be repeated as long as the user needs (see Fig. 2). Successively, the operator can update the fuzzy thresholds according to the results of the ROI statistics analysis and/or his/her visual interpretation of the SAR images.

\subsection{Computation of the membership degree to the set of flooded areas}

Once the set of fuzzy parameters has been selected, the program begins to compute the degree of membership to the set of flooded pixels based on SAR data (see Fig. 2). For this purpose, the standard Z-function is first applied to image1 to compute the degree of membership to the set of low backscattering areas (i.e., of dark image pixels, see Fig. 3, left panel). Then, the set of homogenous areas (see Sect. 2.2) is created and the weighted average rule (see Sect. 2.2) is applied to the aforementioned two fuzzy sets to produce the fuzzy set of the dark and homogeneous pixels. On the average, the largest weight is given to the set of dark areas.

To generate the fuzzy set of pixels involved in the double bounce backscattering enhancement, three applications of the standard $\mathrm{S}$-function to the pixel by pixel difference (in $\mathrm{dB}$ units) between image- 1 and image- 2 are performed. These applications are associated with the three land cover classes potentially involved in the double bounce backscattering, i.e., agricultural, urban and forested areas. Note that this part of the algorithm is actually executed only if image- 2 is used. In this case, the degree of membership to the set of flooded pixels based on SAR data is determined by the fuzzy union (see Sect. 2.2) of the set of dark and homogeneous pixels and of that of pixels involved in the double bounce backscattering enhancement, otherwise it is equal to the former fuzzy set.

At this point, the operator can decide whether to perform the calculation of the distance from the water bodies (see Fig. 2) or not, depending on the temporal requirements. This can be computationally expensive, especially if large SAR scenes are analyzed. If the calculation is enabled, two fuzzy sets are created, i.e., the sets of the pixels close to the water bodies and of the pixels at an altitude not exceeding that of the nearest water pixel (sets (B) and (C) in Sect. 2.2). Then, these sets are combined with that of flooded areas based on SAR data through the weighted average rule, generating the 

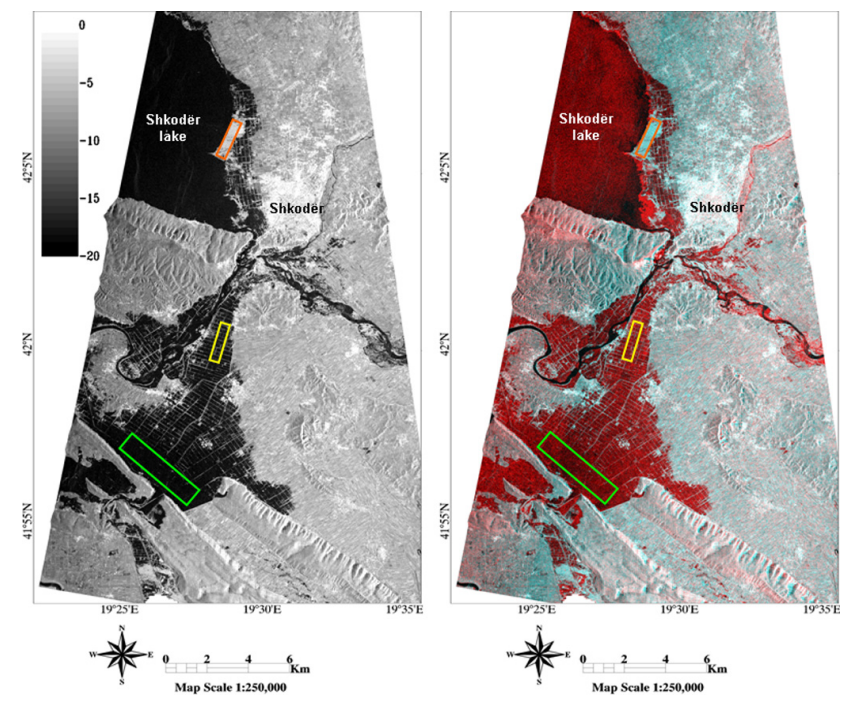

Fig. 3. COSMO-Skymed images of the area of Shkodër (Albany). Left panel: acquisition of 10 January 2010 (the values on the greyscale are in $\mathrm{dB}$ units); right panel: RGB true colour composition of the acquisitions of 7 July 2009 (red) and 10 January 2010 (green and blue). Green, yellow and orange boxes denote three ROIs drawn on the images. White areas correspond to no-data (regions not included in the analysis or areas not imaged by COSMOSkyMed). The urban area of Shkodër is indicated.

fuzzy set of inundated pixels. Also in this case, the largest weight is given to SAR.

The fuzzy set of inundated pixels is further modified in order to account for contextual information through the creation of the sets denoted as (D), (E), (F) in Sect. 2.2. Details on the way we have introduced contextual information in the algorithm can be found in Pierdicca et al. (2008) so that, for the sake of conciseness, we refer the reader to this open access paper. The degrees of membership to the sets (D), (E), (F) are combined with the degree of membership to the fuzzy set of inundated pixels through the weighted average rule, in which the largest weight is given to the latter degree. The final degree of membership resulting from this average is used to generate the map of flooded areas.

\subsection{Generation of the map of flooded areas}

The last part of the program performs the defuzzification of the fuzzy set of flooded areas, followed by a majority analysis and by two editing operations. A simple defuzzification scheme, in which all pixels having a membership degree larger than a threshold value are assigned to the class of flooded areas, is applied. The threshold is determined by means of the Otsu method (Otsu, 1979) that automatically performs histogram shape-based image thresholding, creating the hypothesis that the image to be thresholded (that formed by the degrees of membership of each image pixel, in this case) contains two classes (flooded and non-flooded pixels, in this case). It computes the optimum threshold separating the two classes so that their within-class variances are minimal.

As for the majority analysis, it has the objective of removing residual spurious pixels, thus producing a final map in which flooded and non-flooded areas are homogeneous. It is simply carried out by means of a mobile window, whose dimensions can be selected by the operator (see Fig. 2). The central pixel of the window is classified as flooded if the majority of the pixels belong to this class.

The final step of the program consists of the final editing of the map. Firstly, the algorithm performs an automatic editing operation that basically consists of the detection of the areas possibly involved by shadow/layover effects. This detection is carried out by applying a $3 \times 3$-pixels window to the DEM and computing the standard deviation of the heights within the window. Pixels originally classified as flooded whose standard deviation of the height is larger than a given threshold ( 0.7 , but also in this case we expect to update this value through the analysis of other case studies), are flagged. Note that some SAR processing tools are able to produce maps of layover/shadow using the available orbit information and the DEM of the area. Since the availability of this kind of map is not ensured in operative conditions, we have preferred to resort only to the DEM for this editing step.

The second editing operation is manual and can be optionally performed by the operator to correct the possible errors that he/she finds on the map on the basis of his/her visual interpretation. Errors detectable by a fairly skilled user can be false alarms caused by residual speckle noise or failures in detecting layover/shadow, as well as missed detection due to the presence of wind. The user can draw a ROI on the portion of the map he/she wants to edit and can carry out two different editing actions: (i) inserting new flooded areas not detected by the algorithm; (ii) removing from the ROI the pixels marked as flooded.

\section{Application of the algorithm to the COSMO-SkyMed observations of the Shkodër flood (January 2010)}

Although the OPERA project is intended as a multi-mission demonstration, it pays specific attention to the data provided by the new COSMO-SkyMed mission satellite (Boni et al., 2009) because, as briefly discussed in the introduction, it is particularly useful for operational flood detection. Within the framework of this project, considering also that the Italian Civil Protection was involved in the relief efforts, ASI provided us with a set of observations of the flood that occurred in Albania close to the city of Shkodër, on January 2010. Such an event, which began on 4 January 2010, was caused by heavy rainfall and unusually warm weather that gave rise to snow melting. Moreover, the authorities decided to open the gates of a hydropower station dam to prevent 

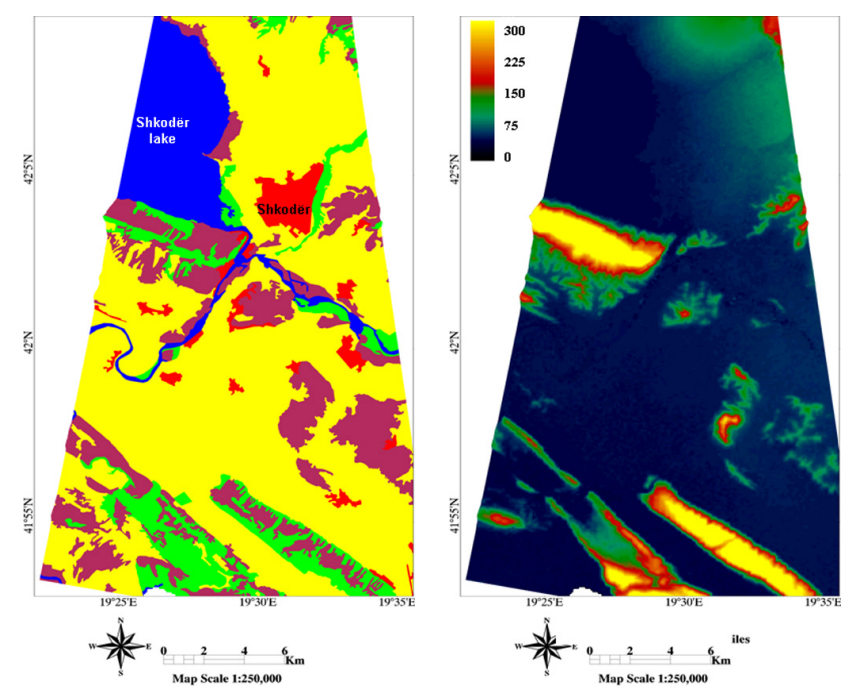

Fig. 4. Land Cover Map (left panel), derived from the Corine 2000 database, and Digital Elevation Model (right panel), derived from the SRTM mission, of the area of Shkodër. For the Land Cover Map, red: urban or built-up areas; green: bare soils; yellow: agricultural areas; maroon: forests; blue: water bodies. For the Digital Elevation Model the stretching is between $0 \mathrm{~m}$ and $300 \mathrm{~m}$ (see the colorbar). White areas correspond to no-data (regions not included in the analysis or areas not imaged by COSMO-SkyMed).

it from breaking. More than 10000 hectares of land were flooded.

To describe the application of our algorithm to a case study and to compare its performance with that yielded by a standard thresholding method, we refer to the CSK acquisition temporarily closest to the peak of the event, i.e., the observation performed on 10 January 2010 (image-1). The image was acquired in Stripmap mode, horizontal polarization, right descending orbit, with an observation angle of $35.9^{\circ}$. An archived image of the same area acquired on 7 July 2009 in Scansar mode, horizontal polarization, right descending orbit, and an observation angle of $22^{\circ}$, was also available (image-2). The images were geocoded by means of appropriate software and exploiting the freely available SRTM (Shuttle Radar Topography Mission) DEM. Then, they were resampled to a resolution of $5 \mathrm{~m}$, and a Frost adaptive filter (Frost et al., 1982) was applied to mitigate the speckle noise. Note that a resolution of $5 \mathrm{~m}$ is degraded with respect to that of the CSK Stripmap products $(3 \mathrm{~m})$, but the Scansar resolution is $30 \mathrm{~m}$ and we have avoided an excessive oversampling of the data acquired on 7 July 2009 . A portion of $4000 \times 6200$ pixels, including the area involved in the flood, has been finally selected.

The image acquired on 10 January 2010 is shown in the left panel of Fig. 3, while the right panel shows a RGB true colour image in which the red channel represents the observation under dry conditions (7 July 2009) while in the other two channels the observation of 10 January 2010 is dis-
Table 2. Mean values of the radar return in the ROIS drawn on Fig. 3.

\begin{tabular}{lcc}
\hline ROI color & $\begin{array}{c}\text { Image-1 }[\mathrm{dB}]- \\
\text { 10 January 2010 }\end{array}$ & $\begin{array}{c}\text { Image-2 }[\mathrm{dB}]- \\
\text { 7 July 2009 }\end{array}$ \\
\hline Green & -19.1 & -14.7 \\
Yellow & -17.9 & -12 \\
Orange & -7 & -13.8 \\
\hline
\end{tabular}

played. Many flooded areas can be visually detected since they appear dark in the left panel of Fig. 3 and red in the right panel. However, it can be noted that also the region of the Shkodër Lake appears red in the right panel because of the presence of wind in this region on 7 July 2009. The area of the Shkodër Lake can be easily detected by looking at the left panel of Fig. 4 showing the LCM that has been derived from the Corine 2000 database.

The area within the orange ROI in Fig. 3 deserves particular attention. It appears bright in the left panel of Fig. 3 and cyan in the right panel of Fig. 3. This indicates that a considerable increase of the backscattering occurred on 10 January 2010 with respect to that measured by CSK on 7 July 2009. This increase is quantified in the last row of Table 2, where it is reported that the mean values of the radar return inside the three ROIs drawn in Fig. 3 for the two days considered in this analysis. It can be noted that the mean value of $\Delta \sigma^{0}$ in the orange ROI is $6.8 \mathrm{~dB}$. In addition, this area is classified as a forest by the LCM (see Fig. 4) and $6.8 \mathrm{~dB}$ represents a value that exceeds the default value of $x_{2}$ for the class of forests that is equal to $5 \mathrm{~dB}$, as reported in Table 1 , suitable for this case study because it refers to an incidence angle of $35^{\circ}$. We can therefore suppose that the area within the orange box was involved in the enhancement of the double bounce backscattering at the time of the inundation. The capability of our algorithm to automatically detect the flood in this area without any supervision will be shown hereafter.

Besides the orange box, other two ROIs are drawn in Fig. 3. Green and yellow boxes include two areas that appear dark in the left panel (probably flooded). For the image acquired on 10 January 2010, the mean values of $\sigma^{0}$ in these two ROIs are equal to $-19.1 \mathrm{~dB}$ (green ROI) and $-17.9 \mathrm{~dB}$ (yellow ROI), as reported in Table 2. These values are consistent with the default value of $x_{1}$ for the fuzzy set of low backscattering areas $(-19 \mathrm{~dB}$, see Table 1$)$ and can therefore be retained.

The verification of the suitability of the default thresholds for the class of low backscattering areas, which basically requires the visual identification of dark areas, is often quite easy and can be carried out also by fairly inexperienced operators. Conversely, checking and eventually modifying the fuzzy thresholds for the classes of agricultural, forested and urban areas are operations that require a certain degree of 
Table 3. Confusion matrix between the classification yielded by our algorithm using the COSMO-SkyMed images acquired on 10 January 2010 and 7 July 2009, and that produced by the histogram thresholding method (only the image of 10 January 2010). Columns represent the result obtained by applying our algorithm.

\begin{tabular}{lcc}
\hline & Non-flooded & Flooded \\
\hline Non-flooded & $93.1 \%$ & $3.2 \%$ \\
Flooded & $6.9 \%$ & $96.8 \%$ \\
Total & $100 \%$ & $100 \%$ \\
\hline
\end{tabular}

Table 4. Same as Table 3, but considering forested areas only.

\begin{tabular}{lcc}
\hline & Non-flooded & Flooded \\
\hline Non-flooded & $94.5 \%$ & $76.3 \%$ \\
Flooded & $5.5 \%$ & $23.7 \%$ \\
Total & $100 \%$ & $100 \%$ \\
\hline
\end{tabular}

skill. We have supposed that an unskilled operator has run our algorithm, so that, to produce the map of inundated areas, we have retained the default threshold values for the three aforementioned classes, thus relying on the electromagnetic models.

For the considered test case, we have allowed the program to calculate the distance from the water bodies. In general, for real-time operative applications, the use of this information is recommended if the time requirements are not very strict, otherwise it can be skipped (unless a map of the distance from the water bodies was already produced by a previous run of the system, thus being already available).

The result of the application of our algorithm to the case study considered is shown in the left panel of Fig. 5. Having assumed that an unskilled operator has run the algorithm, we have not carried out any manual editing to generate this map. In the right panel of Fig. 5 the map obtained by applying a simple histogram thresholding method is shown as a benchmark. The latter map represents the typical outcome of many flood detection methods that search for regions of low backscatter. In this case, the map has been obtained by applying the Otsu method (Otsu, 1979) directly to the CSK image of 10 January 2010.

From a visual comparison between the two maps shown in Fig. 5, the fairly large number of false alarms produced by the thresholding algorithm in the western part of the map can be noted. These false alarms are mostly due to shadowing effects that are not accounted for. Indeed, they occur in a hilly area as can be deduced by looking at the right panel of Fig. 4 that shows the DEM. Pixels affected by shadowing/layover can be flagged by using the DEM of the area and the available orbit information, as previously discussed, The
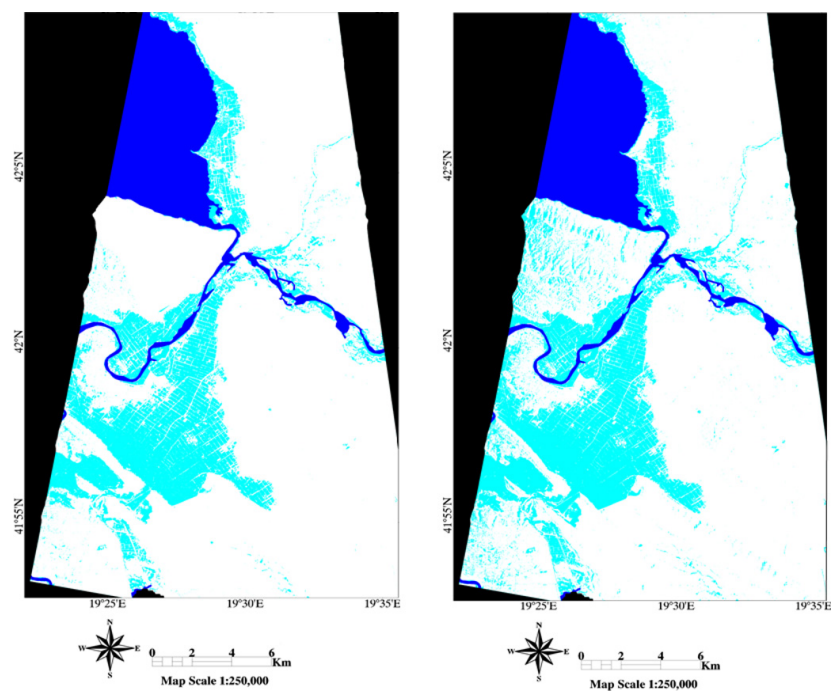

Fig. 5. Map of flooded areas for the Shkodër flood (10 January 2010). Left panel: map produced by our algorithm; right panel: map produced by a histogram thresholding method. White: non-flooded; cyan: flooded; blue: water bodies according to the land cover map; black: no-data.

most significant difference between the maps shown in Fig. 5 regards the area, close to the Shkodër Lake, that is identified by the orange ROI in Fig. 3. According to our classification this area is mostly flooded, whereas the thresholding algorithm assigns this area to the class of non-flooded pixels. This difference is due to the fact that we have introduced the condition of the increase of radar return caused by the double bounce backscattering that may happen in forests. Considering that this area is close to the Shkodër Lake, that its altitude is low (see Fig. 4, right panel) and that it is surrounded by dark pixels (see Fig. 3, left panel), we trust our result.

The differences between the two maps shown in Fig. 5 are quantified in Table 3 in terms of the confusion matrix. The percentages of agreement are high for flooded and nonflooded areas, mainly because most of the agricultural terrains were bare when the flood occurred, so that searching for low backscattering areas has produced fairly good results for this case study. However, if we consider only the forests, the differences are large, as can be noted looking at Table 4, which is analogous to Table 3, but for forests only. In this case the 76.3 percent of pixels that are classified as flooded by our algorithm are assigned to the class of non-flooded areas by the thresholding one.

Ground truth data were not available to validate the classification. However, we have made a visual comparison with a number of independent flood extent estimates, produced using both SAR (TerraSAR-X, Radarsat) and optical (Formosat) imagery, available at the web sites of the MapAction organization (http://mapaction.org/) and of the ReliefWeb project (http://www.reliefweb.int). We have found a general agreement between our map and those available 


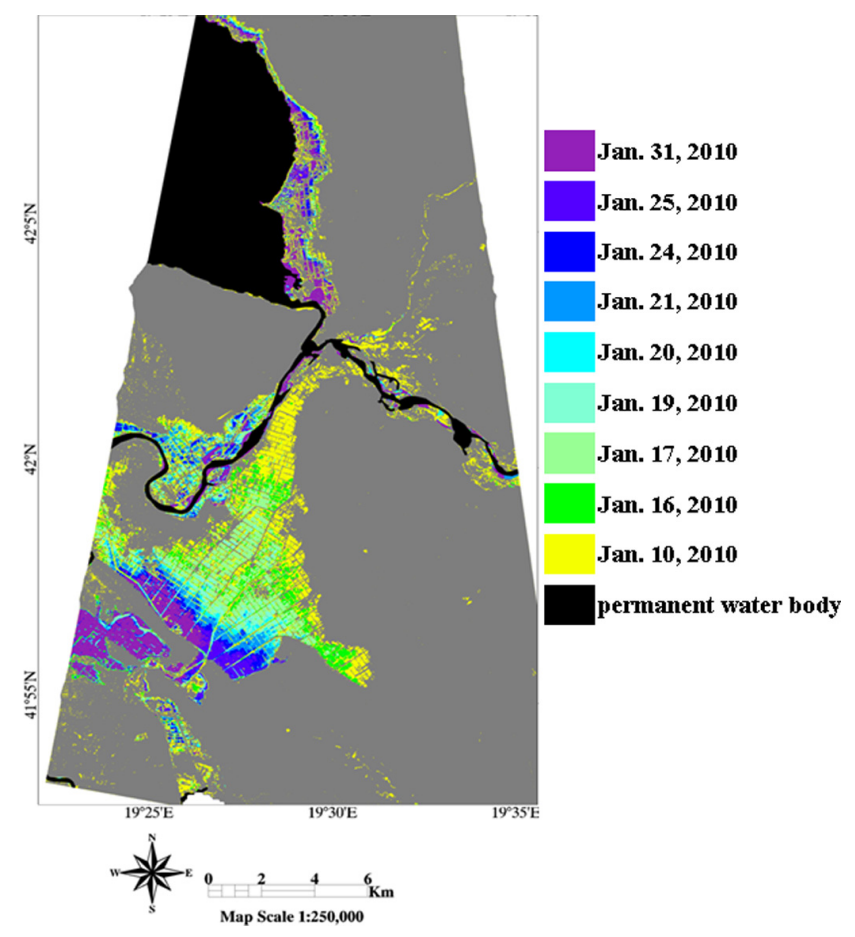

Fig. 6. Multi-temporal map showing the time evolution of the Shkodër flood. It has been produced by applying our algorithm to nine COSMO-SkyMed images whose acquisition dates are reported in the legend. White: no data; gray: non-flooded.

in the aforementioned websites. The most significant difference regards again the area enclosed by the orange ROI in Fig. 3, which is considered as non-flooded by the classifications found on the aforementioned websites.

The COSMO-SkyMed constellation offers a unique opportunity to obtain radar data characterized by short revisit time, thus representing a powerful tool for flood evolution mapping. To give an example of this capability, we have applied our algorithm to nine CSK images of the Shkodër area, acquired during the period 10 January-31 January 2010, provided us by the ASI. In Fig. 6, a multi-temporal map representing time evolution of the Shkodër flood is shown, in which the gradual receding of water can be clearly observed with unprecedented time resolution.

\section{Conclusions}

A new algorithm based on a fuzzy classification approach producing maps of flooded areas using SAR data has been developed and integrated in a prototypical operational system for flood risk management. It allows a user either to quickly produce a classification map, or to perform fairly accurate, but possibly time consuming, flood boundary delineation. The software integrates the theoretical knowledge about the radar return from inundated areas taken into ac- count by means of three well-established electromagnetic models of surface scattering, including hydraulic considerations and textural and contextual information in order to improve the classification accuracy.

The main novelty of the approach is its capability to detect not only open water surfaces, but also forested and agricultural flooded areas, thus allowing a user to cope with situations in which inundation mapping generally represents a difficult task and requires a large amount of supervision in a simple manner. Indeed, if vegetation is composed by cereal crops or forests with moderate biomass, our algorithm permits even unskilled users to automatically generate flood maps exploiting the electromagnetic knowledge introduced in the algorithm through the surface scattering models. In more complex environmental conditions, user interaction is required and allowed by the software.

The proposed algorithm can represent a valuable alternative to simple thresholding methods or segmentation algorithms, thanks to its capacity to integrate different sources of information given by fuzzy logic. Even though it can operate at any frequency between $\mathrm{L}$ and $\mathrm{X}$ bands, particular attention has been paid to the high resolution $\mathrm{X}$ band COSMOSkyMed data. Its application to a COSMO-SkyMed observation of the Shkodër flood occurred on January 2010 has provided fairly good results and improvement with respect to a thresholding procedure has been quantified. A multitemporal map representing the time evolution of the flood has been also shown taking advantage of the short revisit time of COSMO-SkyMed constellation.

At the present status, the choice of the input ancillary data and of the number of land classes the algorithm is capable to manage and of the type of targets for which the fuzzy thresholds are determined, originate from a trade off between system complexity and performance. Future work will concern the update of the fuzzy thresholds, especially those suitable for COSMO-SkyMed measurements, through an integration of the electromagnetic simulations with an empirical data archive that could be built by ad-hoc programming the COSMO-SkyMed acquisitions in specific sites taking into account a larger number of vegetation types and land cover classes than those considered in this study. We plan also to include the roughening due to wind in the model of open water surfaces in order to produce reliable flood extent estimates also in the cases in which the contrast between land and water pixels is not very large. We expect that the aforementioned updates will decrease the number of situations in which user interaction is needed, although the availability of detailed information on environmental conditions (e.g. wind speed and rain data, very detailed land cover maps) of the area involved in the flood may represent a critical aspect.

Acknowledgements. This work has been funded by the Italian Space Agency (ASI) under contract No. I/048/07/0. The authors would like to thank Laura Candela from ASI for her support in gathering COSMO-SkyMed data. The authorization to publish 
results obtained using COSMO-SkyMed data was requested of the ASI on 5 July 2010.

Edited by: F. Guzzetti

Reviewed by: two anonymous referees

\section{References}

Barber, D. G., Hocheim, K. P., Dixon, R., Mosscrop, D. R., and Mcmullan, M. J.: The role of earth observation technologies in flood mapping: A Manitoba case study, Can. J. Remote Sens., 22, 137-143, 1996.

Boni, G., Candela, L., Castelli, F., Dellepiane, S., Palandri, M., Persi, D., Pierdicca, N., Rudari, R., Serpico, S., Siccardi, F., and Versace, C.: The OPERA project: EO-based flood risk management in Italy, Proc. IEEE/IGARSS 2009, Cape Town South Africa, 2009.

Bracaglia, M., Ferrazzoli, P., and Guerriero, L.: A fully polarimetric multiple scattering model for crops, Remote Sens. Environ., 54, 170-179, 1995.

Brakenridge, G. R., Knox, J. C., Paylor, E. D., and Magilligan, F. J.: Radar remote sensing aids study of the great flood of 1993, EOS Transaction American Geophysical Union, 75, 526-527, 1994.

Covello, F., Battazza, F., Coletta, A., Lopinto, E., Fiorentino, C., Pietranera, L., Valentini, G., and Zoffoli, S.: COSMO-SkyMed an existing opportunity for observing the Earth, J. Geodyn., 49, 171-180, 2010

Della Vecchia, A., Ferrazzoli, P., Guerriero, L., Dente, L., Mattia, F., Satalino, G., Strozzi, T., and Wegmüller, U.: Influence of geometrical factors and permittivity models on crop backscattering at C band, IEEE T. Geosci. Remote, 44, 778-790, 2006.

Ferrazzoli, P. and Guerriero, L.: Radar sensitivity to tree geometry and woody volume: a model analysis, IEEE T. Geosci. Remote, 33, 360-371, 1995.

Ferrazzoli, P., Wigneron, J. P., Guerriero, L., and Chanzy, A.: Multifrequency emission of wheat: modeling and applications, IEEE T. Geosci. Remote, 38, 2598-2607, 2000.

Franceschetti, G., Iodice, A., and Riccio, D.: A canonical problem in electromagnetic backscattering from buildings, IEEE T. Geosci. Remote, 40, 1787-1801, 2002.

Frost, V. S., Stiles, J. A., Shanmugan, K. S., and Holtzman, J. C.: A model for radar images and its application to adaptive digital filtering of multiplicative noise, IEEE Transaction on Pattern Analysis and Machine Intelligence, 4, 157-166, 1982.

Henry, J. B., Chastanet, P., Fellah, K., and Desnos, Y. L.: ENVISAT multi-polarised ASAR data for flood-mapping, Int. J. Remote Sens., 27, 1921-1929, 2006.

Hess, L. L., Melack, J. M., and Simonett, D. S.: Radar detection of flooding beneath the forest canopy: A review, Int. J. Remote Sens., 11, 1313-1325, 1990.

Hess, L. L., Melack, J. M., Filoso, S., and Wang, Y.: Delineation of inundated area and vegetation along the Amazon floodplain with the SIR-C synthetic aperture radar, IEEE T. Geosci. Remote, 33, 896-904, 1995.

Horritt, M. S., Mason, D. C., and Luckman, A. J.: Flood boundary delineation from synthetic aperture radar imagery using a statistical active contour model, Int. J. Remote Sens., 27, 1921-1929, 2001.
Karmakar, G. C. and Dooley, L.: A generic fuzzy rule based image segmentation algorithm, Pattern Recogn. Lett., 23, 1215-1227, 2002.

Martinis, S., Twele, A., and Voigt, S.: Towards operational near real-time flood detection using a split-based automatic thresholding procedure on high resolution TerraSAR-X data, Nat. Hazards Earth Syst. Sci., 9, 303-314, doi:10.5194/nhess-9-303-2009, 2009.

Mason, D. C., Speck, R., Devereux, B., Schumann, G., Neal, J. C., and Bates, P. D.: Flood Detection in Urban Areas Using TerraSAR-X, IEEE T. Geosci. Remote, 48, 882-894, 2010.

Nico, G., Pappalepore, M., Pasquariello, G., Refice, A., and Samarelli, S.: Comparison of SAR amplitude vs. coherence flood detection methods- a GIS application, Int. J. Remote Sens., 21, 1619-1631, 2000.

Otsu, N.: A threshold selection method from gray-level histograms, IEEE Transactions on Systems, Man, and Cybernetics, 9, 62-66, 1979.

Pal, S. K. and Rosenfeld, A.: Image enhancement and thresholding by optimization of fuzzy compactness, Pattern Recogn. Lett., 7, 77-86, 1988.

Pierdicca, N., Chini, M., Pulvirenti, L., and Macina, F.: Integrating Physical and Topographic Information into a fuzzy scheme to Map Flooded Area by SAR, Sensors, 8, 4151-4164, 2008.

Pulvirenti, L., Pierdicca, N., and Chini, M.: Analysis of COSMOSkyMed observations of the 2008 flood in Myanmar, Italian Journal of Remote Sensing, 42, 79-90, 2010.

Pulvirenti, L., Chini, M., Pierdicca, N., Guerriero, L., and Ferrazzoli, P.: Flood monitoring using multi-temporal COSMOSkyMed data: image segmentation and signature interpretation, interpretation, Remote Sens. Environ., 115(4), 990-1002, doi:10.1016/j.rse.2010.12.002, 2011.

Rignot, E. J. M. and van Zyl, J. J.: Change detection techniques for ERS-1 SAR data, IEEE T. Geosci. Remote, 31, 896-906, 1993.

Sanyal, J. and Lu, X. X.: Application of remote sensing in flood management with special reference to monsoon Asia: A review, Nat. Hazards, 33, 283-301, 2004.

Schumann, G., Hostache, R., Puech, C., Hoffmann, L., Matgen, P., Pappenberger, F., and Pfister, L.: High-Resolution 3-D Flood Information From Radar Imagery for Flood Hazard Management, IEEE T. Geosci. Remote, 45, 1715-1725, 2007.

Schumann, G., Di Baldassarre, G., and Bates, P. D.: The Utility of Spaceborne Radar to Render Flood Inundation Maps Based on Multialgorithm Ensembles, IEEE T. Geosci. Remote, 47(8), 2801-2807, 2009a.

Schumann, G., Bates, P. D., Horritt, M. S., Matgen, P., and Pappenberger, F.: Progress in integration of remote sensing-derived flood extent and stage data and hydraulic models, Rev. Geophysics, 47, RG4001, doi:10.1029/2008RG000274, 2009b.

Smith, L. C.: Satellite remote sensing of river inundation area, stage, and discharge: a review, Hydrol. Process., 11, 1427-1439, 1997.

Ulaby, F. T. and Dobson, C.: Handbook of Radar Scattering Statistics for Terrain, Artech House, 1989.

Wilson, B. A. and Rashid, H.: Monitoring the 1997 flood in the Red River Valley using hydrologic regimes and RADARSAT imagery, The Canadian Geographer, 1, 100-109, 2005. 